\title{
Inside the fear factor
}

\section{Susanne Ahmari applauds neuroscientist Joseph LeDoux's redefinition of anxiety.}

$\mathrm{S}$ ome 40 million people worldwide have been diagnosed with anxiety disorders. In Anxious, Joseph LeDoux presents a rigorous, in-depth guide to the history, philosophy and scientific exploration of this widespread emotional state. An eminent neuroscientist and author of The Emotional Brain (Simon \& Schuster, 1996) and The Synaptic Self (Viking, 2002), he offers a magisterial review of the role of mind and brain in the generation of both unconscious defensive responses and consciously expressed anxiety.

LeDoux looks first at how our understanding of anxiety has evolved. He starts with ancient etymology (the Greek angh signified constriction) and moves on to Sigmund Freud's view of anxiety as the "root of most if not all mental maladies", and philosopher Søren Kierkegaard's perspective on it as existential, evolving from the dread that stems from freedom of choice. He then lays out the core distinction between fear and anxiety. Fear he defines as anticipation of danger from a physically present threat (a grizzly bear in front of you); anxiety, as anticipation of an uncertain threat (potential predators roaming outside your tent).

But although 'fear' and 'anxiety' are excellent descriptors of conscious feelings, LeDoux shows, they should not be used to describe the unconscious mental processes and neural circuits associated with these emotions. Instead of thinking of those processes as "fear stimuli activate a fear system to produce fear responses", he proposes conceptualizing them as "threat stimuli elicit defense responses via activation of a defensive system". This is a subtle distinction, and LeDoux makes an excellent case that it is an important foundation for rigorous research into the neural underpinnings of the conscious and unconscious processes that subserve anxiety.

He ranges broadly and deeply across molecular neuroscience, psychology and philosophy, yet his methodical approach keeps his argument clear. He starts with a cogent description of threat processing and conditioning based on the 'fight vs flight vs freeze' framework, and moves on to the neural circuits thought to underlie these responses. He gives a clear precis of the possible mental processes behind anxiety disorders (such as impaired ability to discriminate between threat and safety). He then launches into his central thesis: that emotional states of mind such as consciously expressed anxiety are not inherited from our evolutionary

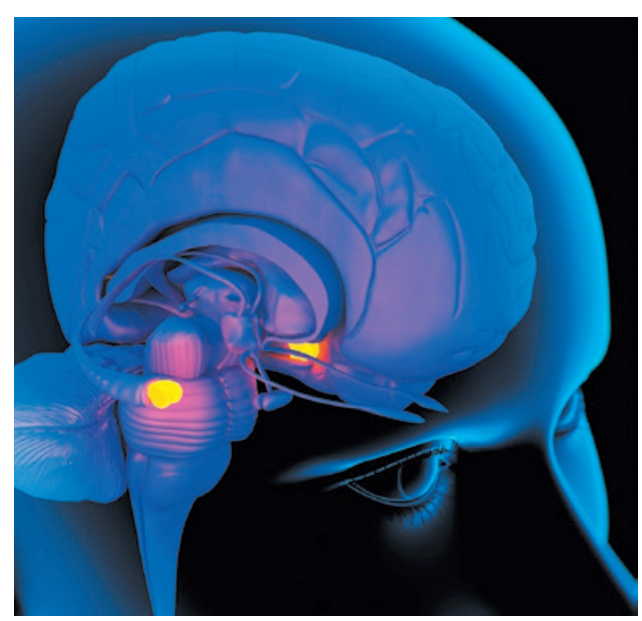

The brain's amygdalae (yellow) are key to anxiety.

ancestors. He argues that decades of animal research have failed to show that animals can consciously express feeling-states in part because of their limited prefrontal cortical development and lack of verbal language. He does not rule out the possibility that animals can consciously feel emotions, but states that "it is not sufficient to provide evidence ... that the behavior in question is consistent with the existence of a conscious experience. One also has to show that the behavior cannot be accounted for by processes that work nonconsciously." A dog may look ecstatic when given a meaty bone, but it is difficult to prove scientifically that it feels what we think of as ecstasy.

The clinical importance of this distinction becomes clear in subsequent chapters, in which the unconscious and conscious brain processes involved in expressing fear and anxiety are ascribed to distinct neural circuits that may have different roles in the pathology and treatment of anxiety disorders. For

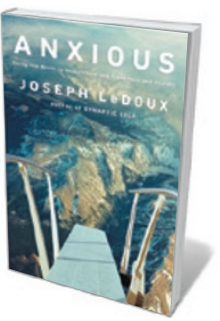

Anxious: Using the Brain to Understand and Treat Fear and Anxiety JOSEPH LEDOUX Viking: 2015. example, when a hiker encounters a snake, information is rapidly and unconsciously sent from the eyes through the sensory thalamus to the amygdala, which can trigger the hiker to freeze before she is aware of the problem. In a slower process, the visual cortex receives the same information from the thalamus, leading to conscious awareness and identification of the snake. In people with anxiety disorders such as post-traumatic stress disorder, the unconscious rapid path to the amygdala may be too strong, leading to perception of threats when none exist.

LeDoux's views jibe with those of many researchers investigating the underpinnings of anxiety disorders, but they are not always acknowledged in the media and elsewhere. Importantly, Anxiety highlights that, although it may not be possible to study the verbally based human emotional experience using animal models, wise use of those models is crucial for progress towards treatments. They remain our only direct window into the molecules, cells and circuits that guide emotions.

Neuroscientists, psychologists, philosophers and psychiatrists will find this exquisitely referenced book particularly useful. It is also a must-read for young investigators, and anyone perusing the footnotes will be rewarded with an insider's view of the state and evolution of anxiety research. LeDoux's charming personal asides give an impression of having a conversation with a world expert.

LeDoux ends on a high note, describing how cutting-edge research on the neural substrates of anxiety is being translated into new approaches for psychiatric treatment. $\mathrm{He}$ discusses, for example, the use of drugs that modulate glutamate-based synaptic transmission to aid exposure therapy for conditions including phobias - a clinical improvement discovered through studies of threat-learning in rats. He also proposes adaptations to therapy protocols that could improve the efficacy of existing treatment. He suggests adjusting the timing of exposure-therapy sessions to maximize consolidation of new learning; sessions scheduled at night, for example, would allow that to happen during sleep without interference from the events of the day.

Such ideas are unproven, and potentially difficult to translate into practice given current constraints on mental-health clinics and care providers. But they are a good example of the transformative potential of cross-talk between basic neuroscience researchers and clinicians. It is only through such synergistic collaborations that we will make significant advances in the treatment of anxiety.

Susanne Ahmari is director of the Translational OCD Laboratory at the University of Pittsburgh in Pennsylvania. She integrates cutting-edge neuroscience with clinical studies to develop treatments. e-mail:ahmarise@upmc.edu 